\title{
Extremal Values of Randić Index among Some Classes of Graphs
}

\author{
Ali Ghalavand $\left(\mathbb{D},{ }^{1}\right.$ Ali Reza Ashrafi $\left(\mathbb{D},{ }^{1}\right.$ and Marzieh Pourbabaee $\mathbb{D}^{2}$ \\ ${ }^{1}$ Department of Pure Mathematics, Faculty of Mathematical Sciences, University of Kashan, Kashan 87317-53153, Iran \\ ${ }^{2}$ Department of Applied Mathematics, Faculty of Mathematical Sciences, University of Kashan, Kashan 87317-53153, Iran \\ Correspondence should be addressed to Ali Ghalavand; alighalavand@grad.kashanu.ac.ir
}

Received 18 April 2021; Accepted 31 May 2021; Published 14 June 2021

Academic Editor: Roslan Hasni

Copyright (c) 2021 Ali Ghalavand et al. This is an open access article distributed under the Creative Commons Attribution License, which permits unrestricted use, distribution, and reproduction in any medium, provided the original work is properly cited.

Suppose $G$ is a simple graph with edge set $E(G)$. The Randić index $R(G)$ is defined as $R(G)=\sum_{u v \in E(G)}\left(1 / \sqrt{\operatorname{deg}_{G}(u) \operatorname{deg}_{G}(v)}\right)$, where $\operatorname{deg}_{G}(u)$ and $\operatorname{deg}_{G}(v)$ denote the vertex degrees of $u$ and $v$ in $G$, respectively. In this paper, the first and second maximum of Randić index among all $n$-vertex $c$-cyclic graphs was computed. As a consequence, it is proved that the Randić index attains its maximum and second maximum on two classes of chemical graphs. Finally, we will present new lower and upper bounds for the Randić index of connected chemical graphs.

\section{Mathematical Notions and Notations}

In this section, we first describe some mathematical notions that will be kept throughout. A pair $G=(V, E)$ in which $V$ is a finite nonempty set and $E$ is a subset of 2-elements subsets of $V$ is called a simple graph. Throughout this paper, the term graph means simple graph and the sets $V=V(G)$ and $E=E(G)$ in definition of $G$ are called the vertex set and edge set of $G$, respectively.

Suppose $G$ is a graph. For simplicity of our argument, an edge $e=\{a, b\}$ in $G$ is simply written as $e=a b$. Choose a vertex $v$ in $G$. The vertex degree of $v, \operatorname{deg}_{G}(v)$, is defined as the number of edges in the form ax. A chemical graph is a graph in which all vertices have degrees less than or equal to 4 [1]. The reason for this name is from quantum chemistry in which it is convenient to model a molecule $M$ in such a way that vertices are used to denote atoms and edges are for chemical bonds.

The set of all vertices adjacent to a vertex $v$ is denoted by $N[v, G]$ and notations $\Delta=\Delta(G), n_{i}=n_{i}(G)$, and $\varepsilon_{i}=\varepsilon_{i}(G)$ are used for the maximum degree, the number of vertices of degree $i$, and the number of edges of degree $i$ in $G$, respectively. The number of edges connecting a vertex of degree $i$ with a vertex of degree $j$ in $G$ is denoted by $m_{i, j}(G)$. A connected $n$-vertex graph $G$ is called to be $c$-cyclic if it has $n+c-1$ edges and the number $c=c(G)$ is said to be the cyclomatic number of $G$.
Suppose $W$ is a nonempty subset of vertices in a graph $G$. The subgraph of $G$ obtained by deleting the vertices of $W$ is denoted by $G-W$, and similarly, if $F \subseteq E(G)$, then the subgraph obtained by deleting all edges in $F$ is denoted by $G-F$. In the case that $W=\{v\}$ or $F=\{x y\}$, the subgraphs $G-W$ and $G-F$ will shortly be written as $G-v$ or $G-x y$, respectively. Furthermore, if $x$ and $y$ are nonadjacent vertices in $G$, then the notation $G+x y$ is used for the graph obtained from $G$ by adding an edge $x y$.

The Randić index of a graph $G$ is defined as

$$
R(G)=\sum_{u v \in E(G)} \frac{1}{\sqrt{\operatorname{deg}_{G}(u) \operatorname{deg}_{G}(v)}} .
$$

This topological index was proposed by Milan Randić [2] under the name "branching index." The Randić index is suitable for measuring the extent of branching of the carbonatom skeleton of saturated hydrocarbons. We encourage the interested readers to consult the books $[3,4]$ for more information on this topic.

\section{Background Materials}

This section aims to briefly review the literature on ordering graphs concerning the Randić index. By referring to Theorems 2.2 and 2.3 in [5], among all $n$-vertex trees, the star $S_{n}$ 
has the minimum Randic index and the path $P_{n}$ attains the maximum Randić index. Caporossi et al. [6] proved that among all 1-cyclic graphs of order $n$, the cycle $C_{n}$ attains the maximum value, and the unicyclic graphs obtained by attaching a pendant path to a vertex of a cycle attain the second maximum Randić index. These are the starting point of the following problem.

Question 1. Find n-vertex c-cyclic graphs with maximum and minimum Randić index.

Shiu and Zhang [7] obtained the maximum value of Randić index in the class of all $n$-vertex chemical trees with $k$ pendants such that $n<3 k-2$. Shi [8] obtained some interesting results for chemical trees with respect to two generalizations of Randić index. Dehghan-Zadeh et al. $[9,10]$ obtained the first and second maximum of Randic index in the class of all $n$-vertex $c$-cyclic graphs when $c=3,4$.

Deng et al. [11] considered various degree mean rates of an edge and gave some tight bounds for the variation of the Randić index of a graph $G$ in terms of its maximum and minimum degree mean rates over its edges. Gutman et al. in a recent interesting paper [12] investigated the connection between Randić index and the degree-based information content of molecular and also general graphs. This connection is based on the linear correlation between Randic index and the logarithm of the multiplicative version of the Randić index.

The aim of this paper is to proceed with Question 1. We will obtain the first and second maximum of Randic index among all $c$-cyclic graphs. This extends some results in $[6,9,10]$.

\section{Five Graph Transformations}

In this section, five graph transformations will be presented which are useful in computing Randić index of graphs. The transformations I and II were introduced in [13].

(1) Transformation $I$. Suppose that $G$ is a graph with a given vertex $w$. In addition, we assume that $P: v_{1}, v_{2}, \ldots, v_{k}$ and $Q: u_{1} u_{2} \ldots u_{l}$ are two paths of lengths $k-1$ and $l-1$, respectively. Let $G_{1}$ be the graph obtained from $G, P$, and $Q$ by attaching edges $v_{1} w$ and $w u_{1}$. Define $G_{2}=G_{1}-v_{1} w+u_{l} v_{1}$. The above-referred graphs are illustrated in Figure 1.

(2) Transformation II. Suppose that $G$ is a graph with given vertices $x$ and $y$ such that $\operatorname{deg}_{G}(x)$, $\operatorname{deg}_{G}(y) \geq 2$ and for all $v \in N[x, G], \operatorname{deg}_{G}(x) \geq 2$. In addition, we assume that $P: v_{1}, v_{2}, \ldots, v_{l}$ and Q: $u_{1}, u_{2}, \ldots, u_{k}$ are two paths of lengths $l-1$ and $k-1$, respectively. Define $G_{1}$ be the graph obtained from $G, P$, and $Q$ by attaching vertices $y v_{1}, u_{1} x$, and $G_{2}=G_{1}-u_{1} x+v_{l} u_{1}$. See Figure 2 for more details.

(3) Transformation III. Suppose that $G$ is a graph with vertices $x, y, w$, and $z$ such that $\{x y, w z\} \subseteq E(G)$. In addition, we assume that $G^{\prime}$ is a trivial graph with vertex set $\{v\}$. Define $G_{1}=G-\{x y\}+\{x v, v y\}$ and $G_{2}=G-\{w z\}+\{w v, v z\}$. The above-referred graphs are illustrated in Figure 3.

(4) Transformation $I V$. Suppose that $G$ is a graph with given vertex $w$ such that $\operatorname{deg}_{G}(w)=\Delta(G) \geq 4$, $N[w, G]=\left\{w_{1}, \ldots, w_{\operatorname{deg}_{G}(w)}\right\} \quad$ and $\operatorname{deg}_{G}\left(w_{1}\right)=$ $\operatorname{deg}_{G}\left(w_{2}\right)=2$. In addition, we assume that $v \in V(G)$, $\operatorname{deg}_{G}(v) \geq 2$, and $v w_{1} \in E(G)$. Define $G^{\prime}=G-$ $w w_{2}+w_{1} w_{2}$. See Figure 4 for more details.

(5) Transformation $V$. Suppose that $G$ is a graph with vertices $x_{1}, x_{2}, x_{3}, x_{4}, x_{5}, x_{6}$, and $w$ such that $\left\{x_{1} x_{2}, x_{2} x_{3}, x_{2} w, x_{4} x_{5}, x_{5} x_{6}\right\} \subseteq E(G), \quad \operatorname{deg}_{G}\left(x_{1}\right)=$ $\operatorname{deg}_{G}\left(x_{2}\right)=\operatorname{deg}_{G}\left(x_{3}\right)=3, \operatorname{deg}_{G}\left(x_{4}\right)=4, \operatorname{deg}_{G}\left(x_{5}\right)=$ 2 , and $\operatorname{deg}_{G}\left(x_{6}\right)=1$ or 2. Define $G^{\prime}=G-$ $\left\{x_{2} w\right\}+\left\{x_{5} w\right\}$. The above-referred graphs are illustrated in Figure 5.

It is well-known that if the derivative $f^{\prime}(x)$ of a continuous function $f(x)$ satisfies $f^{\prime}(x)>0$ on an open interval $(a, b)$, then $f(x)$ is increasing on $(a, b)$.

Lemma 1. The following hold:

(1) Let $G_{1}$ and $G_{2}$ be two graphs satisfying the conditions of Transformation I. If $\operatorname{deg}_{G}(w) \geq 3$ or $\operatorname{deg}_{G}(w)=2$ and $\min _{u w \in E(G)} \operatorname{deg}_{G}(u) \leq 168$ or $\operatorname{deg}_{G}(w)=1$ and $\min _{u w \in E(G)} \operatorname{deg}_{G}(u) \leq 30$, then $R\left(G_{2}\right)>R\left(G_{1}\right)$.

(2) Let $G_{1}$ and $G_{2}$ be two graphs satisfying the conditions of Transformation II and let $\operatorname{deg}_{G}(x)=a$, $N[x, G]=\left\{h_{1}, \ldots, h_{a}\right\}, \quad \operatorname{deg}_{G}\left(h_{i}\right)=d_{i}, \quad \operatorname{deg}_{G}\left(h_{i}\right)=$ $d_{i}, 1 \leq i \leq a, d_{i} \leq d_{i+1}, 1 \leq i \leq a-1$. If $a \geq 5$ or $a=4$ and $d_{2} \leq 20$ or $a=3$ and $d_{2} \leq 6$ or $a=2$ and $d_{2} \leq 5$, then $R\left(G_{2}\right)>R\left(G_{1}\right)$.

(3) Let $G_{1}$ and $G_{2}$ be two graphs as shown in Transformation III.

(a) If $\operatorname{deg}_{G}(x), \operatorname{deg}_{G}(y) \geq 3$, and $\operatorname{deg}_{G}(z) \in\{1,2\}$, then $R\left(G_{2}\right)>R\left(G_{1}\right)$

(b) If $\operatorname{deg}_{G}(x)=2, \operatorname{deg}_{G}(w) \geq 3$, and $\operatorname{deg}_{G}(z)=1$, then $R\left(G_{2}\right)>R\left(G_{1}\right)$

(c) If $\operatorname{deg}_{G}(x)=2$ and $\operatorname{deg}_{G}(w)=2$, then $R\left(G_{2}\right)=R\left(G_{1}\right)$

(4) Let $G$ and $G^{\prime}$ be two graphs satisfying the conditions of Transformation $I V$. Then, $R\left(G^{\prime}\right) \geq R(G)$.

(5) Let $G$ and $G^{\prime}$ be two graphs satisfying the conditions of Transformation $V$. Then, $R\left(G^{\prime}\right)>R(G)$.

Proof

(1) Let $x=\operatorname{deg}_{G}(w), N[w, G]=\left\{l_{1}, \ldots, l_{x}\right\}, d_{G}\left(l_{i}\right)=d_{i}$, $1 \leq i \leq x, \quad \min _{i=1}^{x} d_{i}=d_{1}, \quad$ and $k, l \geq 2$. Then, by definition, 


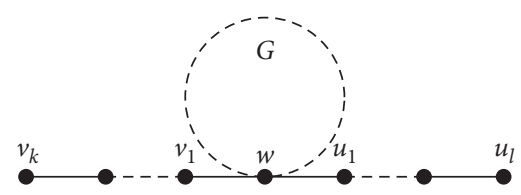

(a)

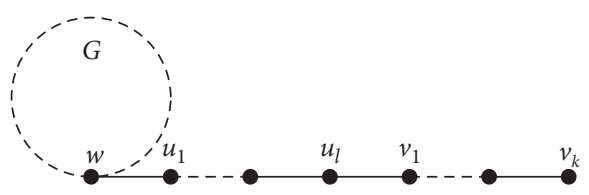

(b)

Figure 1: The graphs (a) $G_{1}$ and (b) $G_{2}$ in Transformation I.

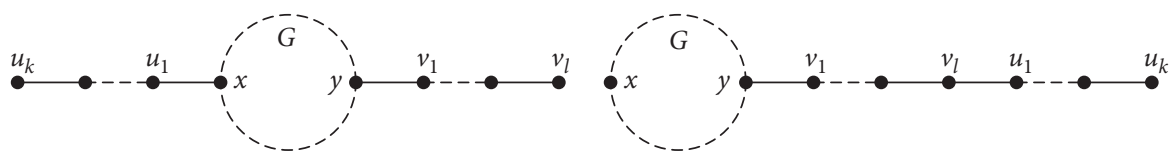

(a)

(b)

Figure 2: The graph (a) $G_{1}$ and (b) $G_{2}$ in Transformation II.

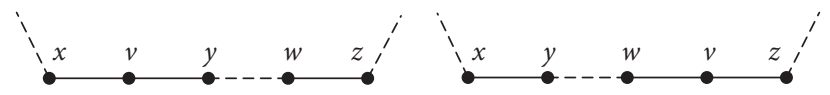

(a)

(b)

FIgURE 3: The graph (a) $G_{1}$ and (b) $G_{2}$ in Transformation III.

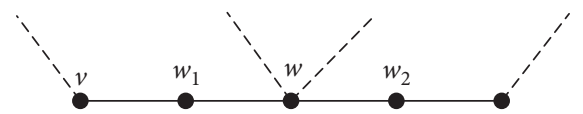

(a)

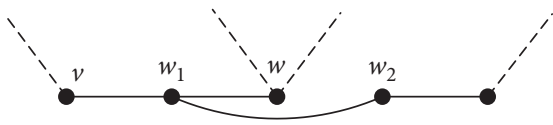

(b)

Figure 4: The graph (a) $G$ and (b) $G^{\prime}$ in Transformation IV.

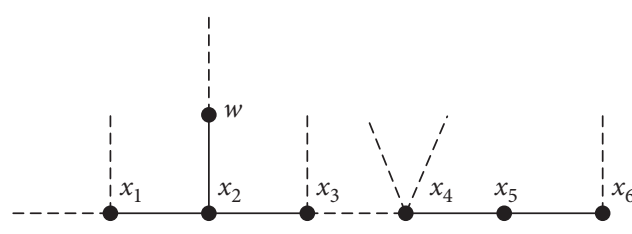

(a)

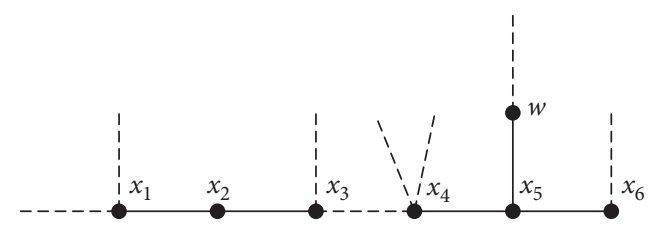

(b)

Figure 5: The graph (a) $G$ and (b) $G^{\prime}$ in Transformation V.

$$
\begin{aligned}
R\left(G_{2}\right)-R\left(G_{1}\right) & =\left(\frac{1}{\sqrt{2(x+1)}}+1+\sum_{i=1}^{x} \frac{1}{\sqrt{d_{i}(x+1)}}\right)-\left(\frac{2}{\sqrt{2(x+2)}}+\frac{1}{\sqrt{2}}+\sum_{i=1}^{x} \frac{1}{\sqrt{d_{i}(x+2)}}\right) \\
& \geq\left[\frac{1}{\sqrt{2}}+\frac{1}{\sqrt{d_{1}}}\right]\left[\frac{1}{\sqrt{x+1}}-\frac{1}{\sqrt{x+2}}\right]-\frac{1}{\sqrt{2}} \frac{1}{\sqrt{x+2}}+1-\frac{1}{\sqrt{2}} .
\end{aligned}
$$

Now, by Equality (2),

$R\left(G_{2}\right)-R\left(G_{1}\right) \geq(1 / \sqrt{2})[(1 / \sqrt{x+1})-(1 / \sqrt{x+2})]$ $+(1 / \sqrt{2})(1 / \sqrt{x+2})+1-(1 / \sqrt{2})>0.013$, for $x \geq 3$, $R\left(G_{2}\right)-R\left(G_{1}\right) \geq 0.0000024$, for $x=2$ and $d_{1} \leq 168$, and $R\left(G_{2}\right)-R\left(G_{1}\right) \geq 0.000086$, for $x=1$ and $d_{1} \leq 30$. The proof of other cases of $k$ and $l$ is similar, and we omit them.

(2) Let $\operatorname{deg}_{G}(x)=a, N[x, G]=\left\{h_{1}, \ldots, h_{a}\right\}, \operatorname{deg}_{G}\left(h_{i}\right)=$ $d_{i}, 1 \leq i \leq a, d_{i} \leq d_{i+1}, 1 \leq i \leq a-1$ and $k, l \geq 2$. Then, by definition, 


$$
\begin{aligned}
R\left(G_{2}\right)-R\left(G_{1}\right) & =\left(1+\sum_{i=1}^{a} \frac{1}{\sqrt{d_{i} a}}\right)-\left(\frac{1}{\sqrt{2(a+1)}}+\frac{1}{\sqrt{2}}+\sum_{i=1}^{a} \frac{1}{\sqrt{d_{i}(a+1)}}\right) \\
& \geq 1-\frac{1}{\sqrt{2(a+1)}}-\frac{1}{\sqrt{2}}+\left[\frac{1}{\sqrt{d_{1}}}+\frac{1}{\sqrt{d_{2}}}\right]\left[\frac{1}{\sqrt{a}}-\frac{1}{\sqrt{a+1}}\right] .
\end{aligned}
$$

And, by last equality,

$R\left(G_{2}\right)-R\left(G_{1}\right)>0.0042$ for $a \geq 5, R\left(G_{2}\right)-R\left(G_{1}\right)$ $>0.00027$ for $a=4$ and $d_{2} \leq 20, R\left(G_{2}\right)-R\left(G_{1}\right)>$ 0.0024 for $a=3$ and $d_{2} \leq 6$, and $R\left(G_{2}\right)-R\left(G_{1}\right)>$
0.0007 for $a=3$ and $d_{2} \leq 5$. The proof of other cases of $k$ and $l$ is similar, and we omit them.

(3) Suppose $\operatorname{deg}_{G}(x)=s, \operatorname{deg}_{G}(y)=r, \operatorname{deg}_{G}(w)=l$, and $\operatorname{deg}_{G}(z)=t$. To prove $(\mathrm{a})$, we note that

$$
\begin{aligned}
R\left(G_{2}\right)-R\left(G_{1}\right) & =\left(\frac{1}{\sqrt{s r}}+\frac{1}{\sqrt{2 l}}+\frac{1}{\sqrt{2 t}}\right)-\left(\frac{1}{\sqrt{2 s}}+\frac{1}{\sqrt{2 r}}+\frac{1}{\sqrt{l t}}\right) \\
& =\left(\frac{1}{\sqrt{s r}}-\frac{1}{\sqrt{2 s}}-\frac{1}{\sqrt{2 r}}\right)+\left(\frac{1}{\sqrt{2 l}}-\frac{1}{\sqrt{2 t}}-\frac{1}{\sqrt{l t}}\right)>-0.48+0.50>0
\end{aligned}
$$

To prove (b), we first calculate the difference between $R\left(G_{2}\right)$ and $R\left(G_{1}\right)$.

$R\left(G_{2}\right)-R\left(G_{1}\right)=\left(\frac{1}{\sqrt{2}}+\frac{1}{\sqrt{2 l}}\right)-\left(\frac{1}{2}+\frac{1}{\sqrt{l}}\right)$.

Let $h(x)=(1 / \sqrt{2 x})-(1 / \sqrt{x})$, for $x \in(0, \infty)$. Then, $h$ is increasing on $(0, \infty)$, and hence by equation (5), $R\left(G_{2}\right)-R\left(G_{1}\right)>0.038$. For the proof of (c), it is enough to notice that $m_{i, j}\left(G_{1}\right)=$ $m_{i, j}\left(G_{2}\right), 1 \leq i \leq j \leq n-1$. Thus, $R\left(G_{2}\right)=R\left(G_{1}\right)$, as desired.

(4) Suppose that $\operatorname{deg}_{G}(v)=s, \quad \operatorname{deg}_{G}(w)=q$, and $\operatorname{deg}_{G}\left(w_{i}\right)=d_{i}, 2 \leq i \leq q$. Then,

$$
\begin{aligned}
R\left(G^{\prime}\right)-R(G) & =\left(\frac{1}{\sqrt{3 s}}+\frac{1}{\sqrt{3(q-1)}}+\frac{1}{\sqrt{6}}+\sum_{i=3}^{q} \frac{1}{\sqrt{(q-1) d_{i}}}\right)-\left(\frac{1}{\sqrt{2 s}}+\frac{2}{\sqrt{2 q}}+\sum_{i=3}^{q} \frac{1}{\sqrt{q^{d_{i}}}}\right) \\
& \geq\left(\frac{2}{\sqrt{6}}+\frac{1}{\sqrt{3(q-1)}}+\frac{q-2}{\sqrt{(q-1) q}}\right)-\left(\frac{1}{\sqrt{4}}+\frac{2}{\sqrt{2 q}}-\frac{q-2}{\sqrt{q^{2}}}\right)>0.02,
\end{aligned}
$$

as desired.

(5) Suppose that $\operatorname{deg}_{G}\left(x_{6}\right)=r$. Then, by definition,

$$
R\left(G^{\prime}\right)-R(G)=\left(\frac{2}{\sqrt{9}}+\frac{1}{\sqrt{8}}+\frac{1}{\sqrt{2 r}}\right)-\left(\frac{2}{\sqrt{6}}+\frac{1}{\sqrt{12}}+\frac{1}{\sqrt{3 r}}\right)>0.0068
$$

Hence, the result.

For a graph $G$, its first Zagreb index $M_{1}(G)$ is defined as $M_{1}(G)=\sum_{v \in V(G)} \operatorname{deg}_{G}(v)^{2}$.

Lemma 2 (see [14]). If $G$ is a connected graph with $n$ vertices and $m$ edges, then
$\varepsilon_{1}(G)=4 m-M_{1}(G)+\sum_{i=3}^{2 n-4} \varepsilon_{i}(G)(i-2)$

$\varepsilon_{2}(G)=M_{1}(G)-3 m-\sum_{i=3}^{2 n-4} \varepsilon_{i}(G)(i-1)$ 
Theorem 1. Let $G$ be a connected graph with $n \geq 3$ vertices and $m$ edges.

(1) If $\Delta(G) \leq 3$ or $\left(\Delta(G) \leq 11\right.$ and $m_{1,4}(G)=m_{2,3}$ $(G)=0), \quad$ then $\quad R(G) \geq\left(4 m-M_{1}(G) / \sqrt{2}\right)+$ $\left(M_{1}(G)-3 m / 2\right)$. The equality holds if and only if $G \cong P_{n}$.

(2) If $\Delta(G) \leq 3$ and $m_{2,2}(G)=m_{1,4}(G)=m_{2,3}(G)=0$ or $\left(\Delta(G) \leq 5\right.$ and $m_{2,2}(G)=m_{1,4}(G)=m_{2,3}(G)=m_{1,5}$ $\left.(G)=m_{2,4} \quad(G)=m_{3,3}(G)=0\right)$, then $R(G) \geq$ $\left(4 m-M_{1}(G) / \sqrt{2}\right)+\left(M_{1}(G)-3 m / \sqrt{3}\right), \quad$ with equality if and only if $G \cong S_{4}$.

Proof. By definition,

$$
\begin{aligned}
R(G)= & \sum_{1 \leq i \leq j \leq n-1} m_{i, j} \frac{1}{\sqrt{i j}} \geq m_{1,2} \frac{1}{\sqrt{2}}+m_{1,3} \frac{1}{\sqrt{3}}+m_{2,2} \frac{1}{2} \\
& +\sum_{i=3}^{2 n-4} \varepsilon_{i}(G) \frac{1}{\Delta(G)} .
\end{aligned}
$$

1. The last equality for $\Delta(G) \leq 3$ gives

$$
\begin{aligned}
R(G) & \geq m_{1,2} \frac{1}{\sqrt{2}}+\frac{1}{2}\left[m_{1,3}+m_{2,2}\right]+\sum_{i=3}^{4} \varepsilon_{i}(G) \frac{1}{3} \\
& =\frac{1}{\sqrt{2}} \varepsilon_{1}(G)+\frac{1}{2} \varepsilon_{2}(G)+\sum_{i=3}^{4} \varepsilon_{i}(G) \frac{1}{3},
\end{aligned}
$$

$$
\begin{aligned}
R(G) \geq & \frac{1}{\sqrt{2}}\left[4 m-M_{1}(G)+\sum_{i=3}^{4} \varepsilon_{i}(G)(i-2)\right] \\
& +\frac{1}{2}\left[M_{1}(G)-3 m-\sum_{i=3}^{4} \varepsilon_{i}(G)(i-1)\right]+\sum_{i=3}^{4} \varepsilon_{i}(G) \frac{1}{3} \\
= & \frac{4 m-M_{1}(G)}{\sqrt{2}}+\frac{M_{1}(G)-3 m}{2}+\left[\frac{1}{\sqrt{2}}-\frac{1}{2}\right] \sum_{i=3}^{4} \varepsilon_{i}(G)(i-1)-\left[\frac{1}{\sqrt{2}}-\frac{1}{3}\right] \sum_{i=3}^{4} \varepsilon_{i}(G) \\
\geq & \frac{4 m-M_{1}(G)}{\sqrt{2}}+\frac{M_{1}(G)-3 m}{2}+2\left[\frac{1}{\sqrt{2}}-\frac{1}{2}\right] \sum_{i=3}^{4} \varepsilon_{i}(G)-\left[\frac{1}{\sqrt{2}}-\frac{1}{3}\right] \sum_{i=3}^{4} \varepsilon_{i}(G) \\
= & \frac{4 m-M_{1}(G)}{\sqrt{2}}+\frac{M_{1}(G)-3 m}{2}+\left[\frac{1}{\sqrt{2}}-1+\frac{1}{3}\right] \sum_{i=3}^{4} \varepsilon_{i}(G) \\
\geq & \frac{4 m-M_{1}(G)}{\sqrt{2}}+\frac{M_{1}(G)-3 m}{2} .
\end{aligned}
$$

Equality holds if and only if $G \cong P_{n}$. Let $\Delta(G) \leq 11$

and $m_{1,4}(G)=m_{2,3}(G)=0$. Then, by Equality (9), 


$$
\begin{aligned}
R(G) & \geq m_{1,2} \frac{1}{\sqrt{2}}+\frac{1}{2}\left[m_{1,3}+m_{2,2}\right]+\sum_{i=4}^{20} \varepsilon_{i}(G) \frac{1}{11} \\
& =\frac{1}{\sqrt{2}} \varepsilon_{1}(G)+\frac{1}{2} \varepsilon_{2}(G)+\sum_{i=4}^{20} \varepsilon_{i}(G) \frac{1}{11},
\end{aligned}
$$

$$
\begin{aligned}
R(G) & \geq \frac{4 m-M_{1}(G)}{\sqrt{2}}+\frac{M_{1}(G)-3 m}{2}+3\left[\frac{1}{\sqrt{2}}-\frac{1}{2}\right] \sum_{i=4}^{20} \varepsilon_{i}(G)-\left[\frac{1}{\sqrt{2}}-\frac{1}{11}\right] \sum_{i=4}^{20} \varepsilon_{i}(G) \\
& =\frac{4 m-M_{1}(G)}{\sqrt{2}}+\frac{M_{1}(G)-3 m}{2}+\left[\frac{2}{\sqrt{2}}-\frac{3}{2}+\frac{1}{11}\right] \sum_{i=4}^{20} \varepsilon_{i}(G) \\
& \geq \frac{4 m-M_{1}(G)}{\sqrt{2}}+\frac{M_{1}(G)-3 m}{2},
\end{aligned}
$$

with equality if and only if $G \cong P_{n}$.

(2) A similar argument as the case 1, it can be proved that for $\Delta(G) \leq 3$ and $m_{2,2}(G)=m_{1,4}(G)=m_{2,3}$ $(G)=0$,

$$
\begin{aligned}
R(G) & \geq \frac{4 m-M_{1}(G)}{\sqrt{2}}+\frac{M_{1}(G)-3 m}{\sqrt{3}}+\left[\frac{2}{\sqrt{2}}-\frac{3}{\sqrt{3}}+\frac{1}{3}\right] \varepsilon_{4}(G) \\
& \geq \frac{4 m-M_{1}(G)}{\sqrt{2}}+\frac{M_{1}(G)-3 m}{\sqrt{3}},
\end{aligned}
$$

with equality if and only if $G \cong S_{4}$. For $\Delta(G) \leq 5$ and $m_{2,2}(G)=m_{1,4}(G)=m_{2,3}(G)=m_{1,5}(G)=m_{2,4}(G)=m_{3,3}$ $(G)=0$,

$$
\begin{aligned}
R(G) & \geq \frac{4 m-M_{1}(G)}{\sqrt{2}}+\frac{M_{1}(G)-3 m}{\sqrt{3}}+\left[\frac{3}{\sqrt{2}}-\frac{4}{\sqrt{3}}+\frac{1}{5}\right] \sum_{i=5}^{8} \varepsilon_{i}(G) \\
& \geq \frac{4 m-M_{1}(G)}{\sqrt{2}}+\frac{M_{1}(G)-3 m}{\sqrt{3}},
\end{aligned}
$$

with equality if and only if $G \cong S_{4}$. This completes the proof.

Theorem 2 (see [6]). Let $G$ be a graph with $n$ vertices. Then,

$$
R(G)=\frac{n}{2}-\frac{1}{2} \sum_{u v \in E(G)}\left(\frac{1}{\sqrt{\operatorname{deg}_{G}(u)}}-\frac{1}{\sqrt{\operatorname{deg}_{G}(v)}}\right)^{2} .
$$

Lemma 3 (see [15]). If $G$ is a connected graph with $n$ vertices and cyclomatic number $c$, then $n_{1}(G)=2-2 c+\sum_{i=3}^{\Delta(G)}(i-$ 2) $n_{i}$ and $n_{2}(G)=2 c+n-2-\sum_{i=3}^{\Delta(G)}(i-1) n_{i}$.
Corollary 1. Let $G$ be a connected graph with $n$ vertices and cyclomatic number $c$.

(1) If $c=5$, then $n_{1}(G)=\sum_{i=3}^{\Delta(G)}(i-2) n_{i}-8$ and $n_{2}(G)=n+8-\sum_{i=3}^{\Delta(G)}(i-1) n_{i}$

(2) If $c=6$, then $n_{1}(G)=\sum_{i=3}^{\Delta(G)}(i-2) n_{i}-10$ and $n_{2}(G)=n+10-\sum_{i=3}^{\Delta(G)}(i-1) n_{i}$

Define $\Upsilon_{1}(n)=\left\{G \mid n_{3}=8, n_{2}=n-8\right\}, \quad \Upsilon_{2}(n)=$ $\left\{G \mid n_{1}=1, n_{3}=9, n_{2}=n-10\right\}, \Upsilon_{3}(n)=\left\{G \mid n_{3}=10, n_{2}=n-\right.$ $10\}$, and $\Upsilon_{4}(n)=\left\{G \mid n_{1}=1, n_{3}=11, n_{2}=n-12\right\}$. 
Lemma 4. Let $G$ be a connected graph with $n$ vertices, $m$ edges, and cyclomatic number $c$.

(1) Suppose $n_{1}=0$ and $0<n_{i}<n$ for some $3 \leq i \leq n-1$. Then, $m_{i, i}(G) \leq n_{i}(G)-2+c$.

(2) Suppose $n_{1} \geq 1$ and $0<n_{i}<n$ for some $3 \leq i \leq n-1$. Then, $m_{i, i}(G) \leq n_{i}(G)-1+c$.
Proof. Since $n_{1}=0, c(G-v) \leq c(G)-1$, for all $v \in V(G)$. Now, the proof follows from this fact that $m=n-1+c$. The part (2) is similar.

Let $n$ be a positive integer. Define

$$
\begin{aligned}
& \Omega_{1}(n)=\left\{G \in \Upsilon_{1}(n) \mid m_{3,3}=11, m_{2,3}=2, m_{2,2}=n-9\right\}, \\
& \Omega_{2}(n)=\left\{G \in \Upsilon_{2}(n) \mid m_{3,3}=13, m_{2,3}=1, m_{1,2}=1, m_{2,2}=n-11\right\}, \\
& \Omega_{3}(n)=\left\{G \in \Upsilon_{3}(n) \mid m_{3,3}=14, m_{2,3}=2, m_{2,2}=n-11\right\}, \\
& \Omega_{4}(n)=\left\{G \in \Upsilon_{4}(n) \mid m_{3,3}=16, m_{2,3}=1, m_{1,2}=1, m_{2,2}=n-13\right\} .
\end{aligned}
$$

If $G_{i} \in \Omega_{i}(n) \quad$ for $\quad 1 \leq i \leq 4$, then $R\left(G_{1}\right)=$ $(1 / 2) n-(5-2 \sqrt{6} / 6), R\left(G_{2}\right)=(1 / 2) n-(7-(\sqrt{6}+3 \sqrt{2}) /$ 6), $R\left(G_{3}\right)=(1 / 2) n-(5-2 \sqrt{6} / 6)$, and $R\left(G_{4}\right)=(1 / 2) n-$ $(7-(\sqrt{6}+3 \sqrt{2}) / 6)$.

Theorem 3. The following hold:

(1) Let $G$ be a connected graph with $n \geq 9$ vertices and cyclomatic number 5. Then, $R(G) \leq(1 / 2) n$ $-(5-2 \sqrt{6} / 6)$, with equality if and only if $G \in \Omega_{1}(n)$.

(2) Let $G$ be a connected graph with $n \geq 11$ vertices and cyclomatic number 6. Then, $R(G) \leq(1 / 2) n$ $-(5-2 \sqrt{6} / 6)$, with equality if and only if $G \in \Omega_{3}(n)$.

Proof

(1) If $n_{1}(G)=0$, then by Corollary 1 and Theorem 2, $R(G) \leq(1 / 2) n-(5-2 \sqrt{6} / 6)$, with equality if and only if $G \in \Omega_{1}(n)$. For $n_{1}(G)=1, R(G) \leq(1 / 2) n$ $-(7-(\sqrt{6}+3 \sqrt{2}) / 6)<(1 / 2) n-(5-2 \sqrt{6} / 6)$. Let $n_{1}(G) \geq 2$. Then, again by Corollary 1 and Theorem 2 , $R(G) \leq(1 / 2) n-(3-2 \sqrt{2} / 2)<(1 / 2) n-(5-$ $2 \sqrt{6} / 6)$.

(2) Proof is similar to the last case, and we omit it.

Remark 1

(1) Let $G$ be a connected graph with $n=8$ vertices and cyclomatic number 5 . Then, $R(G) \leq 4$, with equality if and only if $G$ is a 3-regular graph.

(2) Let $G$ be a connected graph with $n=10$ vertices and cyclomatic number 6 . Then, $R(G) \leq 5$, with equality if and only if $G$ is a 3-regular graph.

For a positive number $c \geq 3$, we define

$$
\begin{aligned}
& \Lambda_{c}^{1}(n)=\left\{G \mid m_{3,3}=3 c-4, m_{2,3}=2, m_{2,2}=n-(2 c-1)\right\} \\
& \Gamma_{c}^{1}(n)=\left\{G \mid m_{3,3}=3 c-2, m_{2,3}=1, m_{1,2}=1, m_{2,2}=n-(2 c+1)\right\} .
\end{aligned}
$$

If $G_{1} \in \Lambda_{c}^{1}(n)$ and $G_{2} \in \Gamma_{c}^{1}(n)$, then $R\left(G_{1}\right)=(1 / 2) n-$ $(5-2 \sqrt{6} / 6)$ and $R\left(G_{2}\right)=(1 / 2) n-(7-(\sqrt{6}+3 \sqrt{2}) / 6)$.

Proposition 1. Let $G$ be a connected graph with $n$ vertices, $m$ edges, and cyclomatic number $c$, where $c \geq 3$ is a positive integer. If $n \geq 2 c-1$ and $n_{1}(G)=0$, then $n_{2}(G) \geq 1$.

Proof. Those are well-known that $c=m-n+1$ and $\sum_{i=1}^{n} n_{i}(G) i=2 m$. Therefore, $2 c=2 n_{2}(G)+\sum_{i=3}^{n} n_{i}(G) i$ $-2 n+2$. Thus, $2 c \geq 2 n_{2}(G)+3\left(n-n_{2}(G)\right)-2 n+2=n-n_{2}$ $(G)+2$, and this implies that $n_{2}(G) \geq n-2 c+2$. Now, since $n \geq 2 c-1, n_{2}(G) \geq 1$.
By Proposition 1 and a similar argument as the proof of Theorem 3, we will have the following general result.

Theorem 4. Let $G$ be a connected graph with $n$ vertices and cyclomatic number $c$, where $c \geq 3$ is a positive integer.

(1) If $n \geq 2 c-1$, then $R(G) \leq(1 / 2) n-(5-2 \sqrt{6} / 6)$, with equality if and only if $G \in \Lambda_{c}^{1}(n)$.

(2) If $n=2 c-2$, then $R(G) \leq(1 / 2) n$, with equality if and only if $G$ is a 3-regular graph.

Let $n$ be a positive number, $\Upsilon_{5}(n)=\left\{G \mid n_{1}=0\right.$, $\left.n_{4}=1, n_{3}=6, n_{2}=n-7\right\} \quad$ and $\Upsilon_{6}(n)=\left\{G \mid n_{1}=0, n_{4}=1\right.$, $\left.n_{3}=8, n_{2}=n-9\right\}$. Define 


$$
\begin{aligned}
& \Omega_{5}(n)=\left\{G \in \Upsilon_{5}(n) \mid m_{4,3}=4, m_{3,3}=6, m_{2,3}=2, m_{2,2}=n-8\right\}, \\
& \Omega_{6}(n)=\left\{G \in \Upsilon_{1}(n) \mid m_{3,3}=10, m_{2,3}=4, m_{2,2}=n-10\right\}, \\
& \Omega_{7}(n)=\left\{G \in \Upsilon_{6}(n) \mid m_{4,3}=4, m_{3,3}=9, m_{2,3}=2, m_{2,2}=n-10\right\}, \\
& \Omega_{8}(n)=\left\{G \in \Upsilon_{3}(n) \mid m_{3,3}=13, m_{2,3}=4, m_{2,2}=n-12\right\} .
\end{aligned}
$$

If $G_{i} \in \Omega_{5}(n)$ for $1 \leq i \leq 4$, then $R\left(G_{1}\right)=(1 / 2) n-(6-$ $(2 \sqrt{3}+\sqrt{6}) / 3), \quad R\left(G_{2}\right)=(1 / 2) n-(5-2 \sqrt{6} / 3), \quad R\left(G_{3}\right)=$ $(1 / 2) n-(6-(2 \sqrt{3}+\sqrt{6}) / 3)$ and $R\left(G_{4}\right)=(1 / 2) n-(5-2$ $\sqrt{6} / 3)$.

Theorem 5. The following hold:

(1) Let $G$ be a connected graph with $n \geq 9$ vertices and cyclomatic number 5. If $G \notin \Omega_{1}(n)$, then $R(G) \leq(1 / 2) n-(6-(2 \sqrt{3}+\sqrt{6}) / 3)$, with equality if and only if $G \in \Omega_{5}(n)$.

(2) Let $G$ be a connected graph with $n \geq 11$ vertices and cyclomatic number 6 . If $G \notin \Omega_{3}(n)$, then $R(G) \leq(1 / 2) n-(6-(2 \sqrt{3}+\sqrt{6}) / 3)$, with equality if and only if $G \in \Omega_{7}(n)$.

Proof

(1) Let $n_{1}(G)=0$ and $G \notin \Omega_{1}(n)$. For $\Delta(G)=3, m_{2,3} \geq 4$ and Corollary 1 and Theorem 2 give $R(G) \leq(1 / 2) n-$ $(5-2 \sqrt{6} / 3)<(1 / 2) n-(6-\quad(2 \sqrt{3}+\sqrt{6}) / 3)$. For $\Delta(G) \geq 4, \quad R(G) \leq(1 / 2) n-(6-(2 \sqrt{3}+\sqrt{6}) / 3)$, with equality if and only if $G \in \Omega_{5}(n)$. If $n_{1}(G) \geq 1$, then $R(G) \leq(1 / 2) n-(7-(\sqrt{6}+3 \sqrt{2}) / 6)<(1 / 2)$ $n-(6-(2 \sqrt{3}+\sqrt{6}) / 3)$.

(2) Proof is similar to Case 1 , and we omit it.

By a simple calculation, one can easily see that Theorem 5 (1) holds for $n=8$ and Theorem 5 (2) holds for $n=10$. On the other hand, Theorems 3 and 5 imply the following result.

Corollary 2. The following hold:

(1) Suppose $n \geq 9$. The connected graphs with cyclomatic number 5 in the sets $\Omega_{1}(n)$ and $\Omega_{5}(n)$ have the first and second maximum Randic index among all n-vertex connected graphs with cyclomatic number 5, respectively.

(2) Suppose $n \geq 11$. The connected graphs with cyclomatic number 6 in the sets $\Omega_{7}(n)$ and $\Omega_{8}(n)$ have the first and second maximum Randic index among all $n$-vertex connected graphs with cyclomatic number 6 , respectively.

Suppose $c \geq 4$ is a positive integer. Define

$$
\begin{aligned}
& \Lambda_{c}^{2}(n)=\left\{G \mid m_{3,3}=3 c-5, m_{2,3}=4, m_{2,2}=n-2 c\right\} \\
& \Gamma_{c}^{2}(n)=\left\{G \mid m_{4,3}=4, m_{3,3}=3 c-9, m_{2,3}=2, m_{2,2}=n-(2 c-2)\right\} .
\end{aligned}
$$

If $H_{1} \in \Lambda_{c}^{2}(n)$ and $H_{2} \in \Gamma_{c}^{2}(n)$, then $R\left(H_{1}\right)=(1 / 2) n-$ $(5-2 \sqrt{6} / 3)$ and $R\left(H_{2}\right)=(1 / 2) n-(6-(2 \sqrt{3}+\sqrt{6}) / 3)$.

Theorem 6. Let $G$ be a connected graph with $n$ vertices and cyclomatic number $c \geq 4$.

(1) If $n \geq 2 c-1$ and $G \notin \Lambda_{c}^{1}(n)$, then $R(G) \leq$ $(1 / 2) n-(6-(2 \sqrt{3}+\sqrt{6}) / 3)$, with equality if and only if $G \in \Gamma_{c}^{2}(n)$.

(2) If $n=2 c-2$ and $G$ is not a 3-regular graph, then $R(G) \leq(1 / 2) n-(6-(2 \sqrt{3}+\sqrt{6}) / 3)$, with equality if and only if $G \in \Gamma_{c}^{2}(n)$.

Proof. The result follows from Proposition 1 and a similar argument as Theorem 3.

We end this section with the following result that follows from Theorems 4 and 6 .
Theorem 7. Let $G$ be a connected graph with $n$ vertices and cyclomatic number $c \geq 4$.

(1) If $n \geq 2 c-1$, then the connected graphs with cyclomatic number $c$ in the sets $\Lambda_{c}^{1}(n)$ and $\Gamma_{c}^{2}(n)$ have the first and second maximum Randic index among all n-vertices connected graphs with cyclomatic number $c$, respectively.

(2) If $n=2 c-2$, then the 3-regular connected graphs and the connected graphs in the set $\Gamma_{c}^{2}(n)$ with cyclomatic number $c$ have the first and second maximum Randic index among all n-vertices connected graphs with cyclomatic number $c$, respectively.

\section{Connected Chemical Graphs}

Let $G$ be a connected chemical graph with $n$ vertices and $m$ edges. By Lemma 3, 


$$
\begin{aligned}
& n_{1}=2 n-2 m+n_{3}+2 n_{4}, \\
& n_{2}=2 m-n-2 n_{3}-3 n_{4},
\end{aligned}
$$

and by Lemma 2 ,

$$
\begin{aligned}
& \varepsilon_{1}(G)=4 m-\left[n_{1}+4 n_{2}+9 n_{3}+16 n_{4}\right]+\varepsilon_{3}(G)+2 \varepsilon_{4}(G)+3 \varepsilon_{5}(G)+4 \varepsilon_{6}(G), \\
& \varepsilon_{2}(G)=n_{1}+4 n_{2}+9 n_{3}+16 n_{4}-3 m-\left[2 \varepsilon_{3}(G)+3 \varepsilon_{4}(G)+4 \varepsilon_{5}(G)+5 \varepsilon_{6}(G)\right]
\end{aligned}
$$

Now, by some calculations, we have

$$
\begin{aligned}
& \varepsilon_{1}(G)=2 n-2 m-2 n_{3}-6 n_{4}+\varepsilon_{3}(G)+2 \varepsilon_{4}(G)+3 \varepsilon_{5}(G)+4 \varepsilon_{6}(G), \\
& \varepsilon_{2}(G)=3 m-2 n+2 n_{3}+6 n_{4}-\left[2 \varepsilon_{3}(G)+3 \varepsilon_{4}(G)+4 \varepsilon_{5}(G)+5 \varepsilon_{6}(G)\right] .
\end{aligned}
$$

Suppose $M_{k}=\left\{m_{i, j} \mid 1 \leq i \leq j \leq 4\right.$ and $\left.i+j=k+2\right\}, \quad 1 \leq$ $k \leq 4$. Since $\varepsilon_{k}=\sum_{x \in M_{k}} x, 1 \leq k \leq 4$,

$$
\begin{aligned}
& m_{1,2}=2 n-2 m-2 n_{3}-6 n_{4}+m_{1,4}+m_{2,3}+2 m_{2,4}+2 m_{3,3}+3 m_{3,4}+4 m_{4,4}, \\
& m_{2,2}=3 m-2 n+2 n_{3}+6 n_{4}-\left[m_{1,3}+2 m_{1,4}+2 m_{2,3}+3 m_{2,4}+3 m_{3,3}+4 m_{3,4}+5 m_{4,4}\right]
\end{aligned}
$$

and since $m_{1,3}=3 n_{3}-m_{2,3}-2 m_{3,3}-m_{3,4}$ and $m_{1,4}=4 n_{4}-$ $m_{2,4}-m_{3,4}-2 m_{4,4}$,

$$
\begin{aligned}
m_{1,2}= & 2 n-2 m-2 n_{3}-2 n_{4}+m_{2,3}+m_{2,4}+2 m_{3,3} \\
& +2 m_{3,4}+2 m_{4,4} \\
m_{2,2}= & 3 m-2 n-n_{3}-2 n_{4}-m_{2,3}-m_{2,4}-m_{3,3} \\
& -m_{3,4}-m_{4,4} .
\end{aligned}
$$

$$
R(G)=\frac{1}{\sqrt{2}} m_{1,2}+\frac{1}{\sqrt{3}} m_{1,3}+\frac{1}{2} m_{1,4}+\frac{1}{2} m_{2,2}+\frac{1}{\sqrt{6}} m_{2,3}+\frac{1}{\sqrt{8}} m_{2,4}+\frac{1}{3} m_{3,3}+\frac{1}{\sqrt{12}} m_{3,4}+\frac{1}{4} m_{4,4} .
$$

By equations (25)-(27), we have

$$
\begin{aligned}
R(G)= & \frac{1}{2}(3-2 \sqrt{2})(m+2 \sqrt{2} n+2 n)-\frac{1}{2}(1-2 \sqrt{3}+2 \sqrt{2}) n_{3} \\
& -(\sqrt{2}-1) n_{4}+\frac{1}{6}(\sqrt{3} \sqrt{2}+3 \sqrt{2}-2 \sqrt{3}-3) m_{2,3}+\frac{1}{4}(3 \sqrt{2}-4) m_{2,4} \\
& +\frac{1}{6}(6 \sqrt{2}-4 \sqrt{3}-1) m_{3,3}+\frac{1}{6}(6 \sqrt{2}-\sqrt{3}-6) m_{3,4}+\frac{1}{4}(4 \sqrt{2}-5) m_{4,4} .
\end{aligned}
$$

Theorem 8. Let $G$ be a connected chemical graph with $n>5$ vertices and $m$ edges. Then,
(1) $R(G) \leq(1 / 2)(3-2 \sqrt{2})(m+2 \sqrt{2} n+2 n), \quad$ with equality if and only if $G \cong P_{n}$ or $C_{n}$ 
(2) $R(G) \geq(1 / 6)(2 \sqrt{2}-3)((\sqrt{3} \sqrt{2}+2 \sqrt{3}-3 \sqrt{2}-$ 3) $n-\sqrt{3} \sqrt{2}-2 \sqrt{3}+3 \sqrt{2}-3 m+3)$
Proof. It is easy to see that $n_{3} \geq(1 / 3)\left(m_{2,3}+2 m_{3,3}+m_{3,4}\right)$ and $n_{4} \geq(1 / 4)\left(m_{2,4}+m_{3,4}+2 m_{4,4}\right)$. Therefore, by equation (28), we have

$$
\begin{aligned}
R(G) \leq & \frac{1}{2}(3-2 \sqrt{2})(m+2 \sqrt{2} n+2 n)-\frac{1}{6}(4-\sqrt{3} \sqrt{2}-\sqrt{2}) m_{2,3} \\
& -\frac{1}{4}(3-2 \sqrt{2}) m_{2,4}-\frac{1}{6}(3-2 \sqrt{2}) m_{3,3}-\frac{1}{12}(11-2 \sqrt{3}-5 \sqrt{2}) m_{3,4} \\
& -\frac{1}{4}(3-2 \sqrt{2}) m_{4,4} \leq \frac{1}{2}(3-2 \sqrt{2})(m+2 \sqrt{2} n+2 n),
\end{aligned}
$$

with equality if and only if $G \cong P_{n}$ or $C_{n}$. On the other hand,

$n_{3}+n_{4} \leq n$ and $m_{2,3}+m_{2,4}+m_{3,3}+m_{3,4}+m_{4,4} \geq n_{3}+n_{4}-1$.

Therefore, by equation (28),

$$
\begin{aligned}
R(G) \geq & \frac{1}{2}(3-2 \sqrt{2})(m+2 \sqrt{2} n+2 n)-(\sqrt{2}-1)\left(n_{3}+n_{4}\right) \\
& +\frac{1}{6}(\sqrt{3} \sqrt{2}+3 \sqrt{2}-2 \sqrt{3}-3)\left(n_{3}+n_{4}-1\right) \\
\geq & \frac{1}{2}(3-2 \sqrt{2})(m+2 \sqrt{2} n+2 n)-\frac{1}{6}(3 \sqrt{2}+2 \sqrt{3}-3-\sqrt{3} \sqrt{2})\left(n_{3}+n_{4}+5-2 \sqrt{3} \sqrt{2}\right) \\
\geq & \frac{1}{6}(2 \sqrt{2}-3)((\sqrt{3} \sqrt{2}+2 \sqrt{3}-3 \sqrt{2}-3) n-\sqrt{3} \sqrt{2}-2 \sqrt{3}+3 \sqrt{2}-3 m+3) .
\end{aligned}
$$

This completes the proof.

\section{Data Availability}

All data generated or analyzed during this study are included in this published article. There are no experimental data in this article.

\section{Conflicts of Interest}

The authors declare that there are no conflicts of interest regarding the publication of this article.

\section{References}

[1] M. Ö. Turaci, "On vertex and edge eccentricity-based topological indices of a certain chemical graph that represents bidentate ligands," Journal of Molecular Structure, vol. 1207, Article ID 127766, 2020.

[2] M. Randić, "On characterization of molecular branching," Journal of the American Chemical Society, vol. 97, pp. 66096661, 1975.

[3] I. Gutman and B. Furtula, Recent Results in the Theory of Randic Index, University of Kragujevac, Kragujevac, Serbia, 2008.

[4] X. Li and I. Gutman, Mathematical Aspects of Randic-type Molecular Structure Descriptors, University of Kragujevac, Kragujevac, Serbia, 2006.
[5] X. Li and Y. Shi, "A survey on the Randić index," MATCH Communications in Mathematical and in Computer Chemistry, vol. 59, no. 1, pp. 127-156, 2008.

[6] G. Caporossi, I. Gutman, P. Hansen, and L. Pavlović, "Graphs with maximum connectivity index," Computational Biology and Chemistry, vol. 27, pp. 85-90, 2003.

[7] W. C. Shiu and L. Z. Zhang, "The maximum Randić index of chemical trees with $k$ pendants," Discrete Mathematics, vol. 309, pp. 4409-4416, 2009.

[8] Y. Shi, "Note on two generalizations of the Randić index," Applied Mathematics and Computation, vol. 265, pp. 10191025, 2015.

[9] T. Dehghan-Zadeh, A. R. Ashrafi, and N. Habibi, "Maximum and second maximum of Randić index in the class of tricyclic graphs," MATCH Communications in Mathematical and in Computer Chemistry, vol. 74, pp. 137-144, 2015.

[10] T. Dehghan-Zadeh, A. R. Ashrafi, and N. Habibi, "Tetracyclic graphs with extremal values of Randić index," Bollettino dell Unione Matematica Italiana, vol. 8, pp. 9-16, 2015.

[11] H. Deng, S. Balachandran, and S. Elumalai, "Some tight bounds for the harmonic index and the variation of the Randic index of graphs," Discrete Mathematics, vol. 342, no. 7, pp. 2060-2065, 2019.

[12] I. Gutman, B. Furtula, and V. Katanić, "Randić index and information," AKCE International Journal of Graphs and Combinatorics, vol. 15, no. 3, pp. 307-312, 2018.

[13] A. Ghalavand and A. R. Ashrafi, "Ordering chemical graphs by Randić and sum-connectivity numbers," Applied Mathematics and Computation, vol. 331, pp. 160-168, 2018. 
[14] A. Ghalavand and A. R. Ashrafi, "Bounds on the entire Zagreb indices of graphs," MATCH Communications in Mathematical and in Computer Chemistry, vol. 81, pp. 371-381, 2019.

[15] A. Ghalavand, A. R. Ashrafi, and I. Gutman, "Extremal graphs for the second multiplicative Zagreb index," Bulletin of the International Mathematical Virtual Institute, vol. 8, no. 2, pp. 369-383, 2018. 